\title{
1 Paradoxes in the logistic equation?
}

2 Jean-Pierre Gabriel ${ }^{1}$, Francis Saucy ${ }^{2}$, Louis-Félix Bersier ${ }^{3,4}$

$3 \quad{ }^{1}$ Department of Mathematics, University of Fribourg, ch. du Musée 23, CH-1700 Fribourg,

$4 \quad$ Switzerland. jean-pierre.gabriel@unifr.ch

$5 \quad 2$ Rue des Châteaux 49, CH-1633 Vuippens, Switzerland. francis.saucy@bluewin.ch

$6{ }^{3}$ Zoological Institute, University of Neuchâtel, Rue Emile-Argand 11, C.P. 2, CH-2007 Neuchâtel,

$7 \quad$ Switzerland. louis-felix.bersier@unine.ch

$8 \quad{ }^{4}$ Chair of Statistics, Department of Mathematics, Swiss Federal Institute of Technology, CH-1015

$9 \quad$ Lausanne, Switzerland

type of article : Short communication CH-2007 Neuchâtel; Switzerland. tel: +41327183006 
1 Abstract : Levins and Ginzburg raised two paradoxes concerning the logistic equation of population growth. None of them received a satisfactory answer within the frame of the original equation. Here, we propose solutions to both paradoxes.

keywords : logistic model, population dynamic, population regulation, Verhulst equation.

The logistic equation of population growth occupies a unique and fascinating position in the development of ecological thinking. Proposed in the first half of the nineteenth century by the Belgian mathematician Pierre-François Verhulst (1838) as a potential solution to the dilemma of Malthusian exponential growth, it was rediscovered and imposed to biologists as a simple model of population self-regulation in the early twentieth century by the American biologist Raymond Pearl and his disciples (Kingsland 1985).

The logistic equation has since inspired and stimulated much ecological work, including modeling, experimental and field research. Nowadays, the concept of carrying capacity can hardly be dissociated from the model. It has also permeated evolutionary biology in the 1950's and the concept of $\mathrm{r} / \mathrm{K}$ selection was named after its parameters in the 1970 's. Finally, its remarkable fitting abilities to early datasets have also contributed to the popularity of the model (Pearl 1925; Feller 1940; Hutchinson 1978). The logistic equation can be found in many biology textbooks and belongs to basic education in ecology. Independently of the status that one gives to this model, it has been and remains a cornerstone of empirical and theoretical ecology.

The logistic equation however also elicited strong attacks and vigorous criticisms, so that ecologists have developed a love-hate relationship with the logistic equation (Olson 1992). Many legitimate criticisms address the phenomenological foundations of the model and its adequacy to describe the real world. They include for example the difficulty in the 
1 interpretation of its parameters (e.g., Olson 1992; Miller et al. 2002), or its unrealistic form

2 of density dependence (e.g., Getz 1996; McCarthy 1997; Courchamp et al. 1999; Etienne

3 2002; Kent et al. 2003). Other criticisms have been raised with regard to internal

4

contradictions. Named hereafter paradoxes, these apparent contradictions challenge the consistency of the model itself, and we shall focus here on this issue. We believe that clarifying such points might help understanding the significance and limitations of this historically and educationally important model. Our aim is to discuss two paradoxes raised respectively by Levins (Hutchinson 1978) and Ginzburg (1992), and we show how they can be solved within the framework of the original formulation of the model.

\section{Levins' paradox}

The usual "ecological" form of the logistic equation is:

$$
\frac{\mathrm{d} N}{\mathrm{~d} t}=r N\left(1-\frac{N}{K}\right) .
$$

The first paradox, raised by Levins, has been exposed in Hutchinson (1978, page 4) as follows: “ $r(\ldots)$ be negative, setting $N>K$, leads to an impossible result, for $\mathrm{d} N / \mathrm{d} t$ is positive and increases indefinitely with $N$ '. Indeed, it can be shown that infinity is even reached in a finite time and, to avoid such a situation, Levins suggested that $r$ should be constrained to non-negative values. If so the paradox disappears, but at the cost of excluding biologically meaningful situations, namely declining populations. Obviously, $r$ has to be negative in sink environments (Hairston et al. 1970; Pulliam 1988). Actually, a more sensible resolution emerges from Verhulst's original formulation (1838):

$$
\frac{\mathrm{d} N}{\mathrm{~d} t}=a N-b N^{2},
$$

where $a$ is the Malthusian growth rate and, as such, has to admit both signs. In the second term added to avoid infinite growth, $b$ is a friction coefficient and therefore strictly 
1 positive. Eq. (1) is derived from Eq. (2) by writing the latter as:

$2 \quad \frac{\mathrm{d} N}{\mathrm{~d} t}=a N\left(1-\frac{N}{a / b}\right)$,

3 and identifying $r=a$ and $K=a / b$. These relations are well known, and can be found in

several textbooks. However, a simple but important consequence that, to our knowledge, has never been pointed out is that $r$ and $K$ must have the same sign since $b=r / K$ is positive. This constraint rules out Levin's case $(r<0$ and $K>0)$ and therefore solves the paradox without limiting the sign of $r$. However, with $r<0, K$ is also negative and cannot be interpreted as a "carrying capacity"; it is easy to see that, if $r<0$, then all the solutions with $N(0) \geq 0$ converge to 0 , as expected in sink environments. We see that this leads to a difficulty in the interpretation of the parameter $K=a / b$ as a "carrying capacity”. A way to avoid this problem is to redefine the carrying capacity as $K_{\infty}$ :

$$
K_{\infty}=\lim _{t \rightarrow \infty} N(t), \text { for } N(0)>0
$$

As a consequence, in the logistic case we have :

$$
K_{\infty}= \begin{cases}a / b=K, & \text { if } a>0, \\ 0, & \text { if } a \leq 0 .\end{cases}
$$

It is worth emphasizing that, while $a$ and $b$ are two independent parameters in Verhulst's equation (Eq. (2)), $K$ depends on $r$ in the usual ecological form (Eq. (1)). To our knowledge, the first apparition of the ecological form goes back to Lotka (1925), with a typo in the formula.

At this stage, it is helpful to distinguish between two versions of the logistic model. We call “constrained” logistic the model derived from Verhulst approach, i.e., with $r=a$, $K=a / b$, and $b$ strictly positive. In the "free" logistic model, $r$ and $K$ are considered independent. A classical derivation (e.g., Begon et al. 1996) of the logistic equation assumes a linear decrease of the per capita growth rate (see Fig. 1a). In this approach, $r$ and 
$1 \quad K$ are implicitly assumed to be both positive and independent. Levins’ paradox appears in

2 the free model with $r$ negative (Fig. 1b), but not in the constrained model (Fig. 1c). It is worth noting that Levin's configuration (Fig. 1b) leads to a positive density-dependence.

\section{Ginzburg's paradox}

Ginzburg (1992) considered incorporating additional mortality into the logistic equation without changing any other aspects of the environment, and proposed two ways to do so. Firstly:

$$
\frac{\mathrm{d} N}{\mathrm{~d} t}=r N\left(1-\frac{N}{K}\right)-\mu N,
$$

where $\mu N(\mu>0)$ is the additional mortality term. The new equilibrium density is $K(r-\mu) / r$ $(\mu<r)$. Ginzburg rejected this approach because it "disagrees with our intuition about unchanging equilibrium”. He claimed that, as the resources do not change, the population with higher mortality might attain the same $\mathrm{K}$, but more slowly. This point is obviously disputable. Like all population equations, the logistic is a conservation or balance equation, i.e., the size variation over a time interval is the difference between input and output over that interval. In any case, it is admissible to act on such an equation by adding a term reflecting increased mortality, e.g., by harvesting a constant fraction of the population per unit of time. If one's intuition is puzzled by the consequences of such a change, one must either adapt or reject the whole model, i.e., the logistic itself. Moreover, our own intuition is not hurt by the decrease of the equilibrium density: with additional mortality, individuals are continuously removed from the population, and thus the population might never reach $K$ again. Such a behaviour is ultimately dictated by the model, and not the intuition. Secondly, Ginzburg proposed to introduce additional mortality in $r$ only:

$$
\frac{\mathrm{d} N}{\mathrm{~d} t}=(r-\mu) N\left(1-\frac{N}{K}\right)=r N\left(1-\frac{N}{K}\right)-\mu N\left(1-\frac{N}{K}\right),
$$


1 which does not change $K$. But Ginzburg stated that the additional term $-\mu N(1-N / K)$

cannot represent mortality since it is positive for $N>K$ (note that it is valid only for $K>0$ i.e. $\mu<r)$.

This paradoxical situation fostered a lively debate on equations of population growth in the issues of September 1992 and February 1993 of TREE. Most of these answers were based on models different from the logistic one and we will not tackle them, because our aim is to solve the paradox internally to the logistic equation. Only Young (1992) and Berryman (1992) replied within the framework of the logistic, but their answers are unsatisfactory. Indeed, Young's and Ginzburg's second approach (Eq. (6)) are mathematically equivalent (set $p=1-\mu / r$ in Eq. (7) of Young 1992), and therefore deserve the same analysis given below. With regard to Berryman, although we share his conclusions (Berryman 1992), we cannot adopt his line of reasoning. He tackled the problem by decomposing the model into two density dependent linear birth and death rates leading to the following form of the logistic equation: $\mathrm{d} N / N \mathrm{~d} t=(n-v N)-(s+\sigma N) ; n$ and $s$ are the intrinsic birth and death rates, $v$ and $\sigma$ the density dependent birth and death coefficients. Such a derivation can be found in contemporary textbooks of ecology (e.g., Gotelli 2001) and was already proposed in Kostitzin (1937, pp. 15). A first difficulty is that the number of parameters is different in Ginzburg's and Berryman's equations, leading to problems with their identification. A more fundamental problem lies in the linear birth term with a negative slope, since it becomes negative for large values of $N$. This is conceptually unsatisfactory since it requires a limitation in the values of $N$, and therefore provides a model different from Verhulst's logistic equation. In our opinion, one should abandon this approach and rather stick to Verhulst's derivation, which does not contain this awkward feature. 
Again, returning to Verhulst framework (Eq. (2)) solves Ginzburg's paradox. In the constrained logistic model, the only way of maintaining $K$ fixed and respecting the equalities $r=a$ and $K=a / b$ is to act on $b$. If $a$ becomes $a-\mu(0<\mu<a$ to preserve $K>0)$ then $b$ must be replaced by $b-\delta(0<\delta<b)$, so that $K=a / b=(a-\mu) /(b-\delta)$. Solving for $\delta$, we get $\delta=\mu b / a$, and Eq. (2) becomes:

$$
\frac{\mathrm{d} N}{\mathrm{~d} t}=(a-\mu) N-(b-\delta) N^{2}=a N-b N^{2}-\mu N+\frac{\mu b}{a} N^{2},
$$

It is now apparent that the paradoxical mortality term of Eq. (6) (i.e., $-\mu N(1-N / K)$ ) is composed of the losses due to increased mortality $-\mu N$ and of $\frac{\mu b}{a} N^{2}$, a correction of the second order friction term. The first term is always negative and the second always positive since $K$, and hence $a$, are positive. Clearly, $\frac{\mu b}{a} N^{2}=\left(\frac{N}{K}\right) \mu N$ is larger than $\mu N$ when $N>K$, and the total contribution is positive. This means that the gain obtained by the reduction in the quadratic friction term overcomes the loss due to the linear mortality term. There is no paradox in this, and we want to illustrate it with an example. Let us consider an animal population with cannibalism (e.g. a hypothetical spider population): let $a$ denote the difference between intrinsic birth and death (cannibalism excluded) rates; the brake $b N^{2}$ is due only to cannibalism, assumed to be proportional to the number of meetings per unit time; a predator is added to the system, and removes $\mu N$ spiders per unit time. If one decrees that the equilibrium density must remain constant, then the only choice (except increasing trivially $a$ ) is to act on $b$. The intensity of cannibalism must be weaken, and the number of individuals saved in this way per unit time is $\delta N^{2}\left(\delta=\frac{\mu b}{a}\right)$. For large values of $N(N>K)$, the number $\delta N^{2}$ of individuals saved from cannibalism overcomes the number $\mu N$ of individuals killed by the additional predator. Clearly, Ginzburg's paradoxical term 
1 does not reflect mortality only. Again, returning to the historical formulation of Verhulst

2 puts a constraint on parameters $r$ and $K$, which also solves Ginzburg's paradox.

\section{Discussion}

We would like to stress some important issues raised in this paper. Firstly, we showed that two paradoxes or apparent contradictions appear only in the free logistic model, but disappear in the constrained one. Other "paradoxes" can be produced, e.g. by increasing $r$ without changing $K$, or by decreasing the carrying capacity with $r<0$ and $K>0$. In both cases, the additional terms have the "wrong" sign. Again, both "paradoxes" do not persist in the constraint model. Secondly, it is important to be aware that the choice of the derivation is not innocuous. The approach proposed in Kostitzin (1937) and used in Berryman’s reply (1992) is an example. In the same vein, following Lotka's (1924) derivation based on Taylor's expansion leads to the same difficulty because there is no constraints on the quadratic term. Thirdly, we believe that the logistic equation should be considered as an abstract object conveying the message that a population may exhibit selfregulation via some implicit density-dependent mechanism modeled in the friction term. The phenomenological identification of the parameters requires additional knowledge in each particular situation. Indeed, this implies describing how the model can be implemented in the real world, i.e. how energy or matter is used, enters and leaves the system (Saucy \& Gabriel 1999). We will address this challenging question in a forthcoming paper. Finally, although our goal is neither to rescue nor to dismiss the model, we observe that the logistic equation is of historical importance and of educational significance. For this reason we think valuable to clarify its conceptual framework and its limitations. We hope that solving these two paradoxes will help reaching this goal. 


\section{References}

2 Begon, M., Harper, J.L. \& Townsend C.R., 1996. Ecology: Individuals, Populations and $3 \quad$ Communities. $3^{\text {rd }}$ edition. Blackwell Science.

4 Berryman, A.A., 1992. Intuition and the logistic equation. Trends Ecol. Evol., 7, 316.

5 Courchamp, F., Clutton-Brock, T. \& Grenfell, B., 1999. Inverse density dependence and the Allee effect. Trends Ecol. Evol., 14, 405-410.

Etienne R., Wertheim B., Hemerik L., Schneider P. \& Powell J., 2002. The interaction between dispersal, the Allee effect and scramble competition affects population dynamics. Ecol. Model.,148, 153-68. 
1 Kingsland, S.E., 1985. Modeling Nature. The University of Chicago Press, Chicago and London.

Lotka, A., 1924. Elements of physical biology. Williams and Wilkins Co., Inc.

Lotka, A., 1925. Meeting on the problem of forecasting city populations with special reference to New York city. J. Am. Stat. Assoc., 20, 569-570.

McCarthy, M.A., 1997. The Allee effect, finding mates and theoretical models. Ecol 


\section{Figure legend}

2 Fig. 1. Per capita growth rate as a function of population density $N$ for the logistic model.

3 a) Usual configuration with $r$ and $K$ positive; $K_{\infty}$ as a new definition of the carrying

4 capacity (see Eq. 4). b) Levins’ situation leading to an inverse density dependence. c)

5 Constrained model with $r$ negative. 


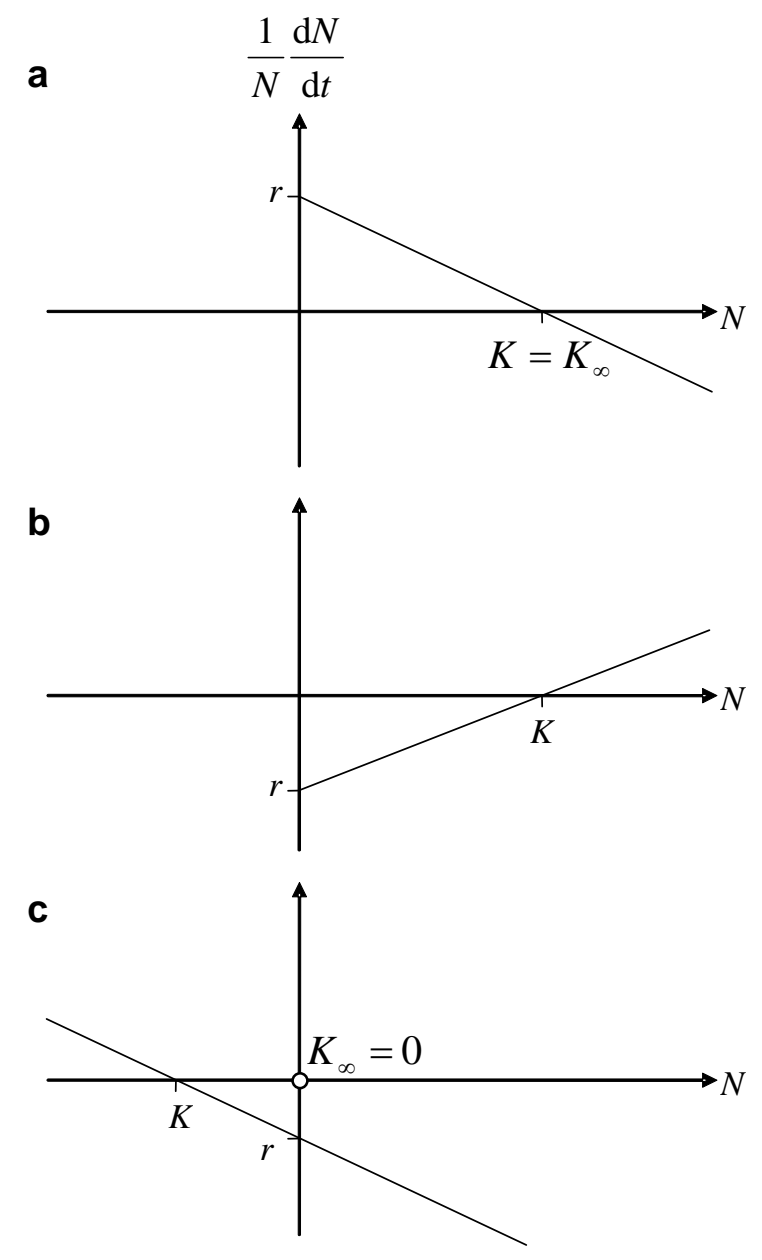

